Gut and Liver, Vol. 12, No. 6, November 2018, pp. 674-681

\title{
Development of a Novel Endoscopic Scoring System to Predict Relapse after Surgery in Intestinal Behçet's Disease
}

\author{
Jung Won Park, Yehyun Park, Soo Jung Park, Tae II Kim, Won Ho Kim, and Jae Hee Cheon \\ Department of Internal Medicine and Institute of Gastroenterology, Yonsei University College of Medicine, Seoul, Korea
}

Background/Aims: The cumulative surgery rate and postoperative relapse of intestinal Behçet's disease (BD) have been reported to be high. This study aimed to establish a scoring system based on follow-up endoscopic findings that can predict intestinal BD recurrence after surgery. Methods: Fifty-four patients with intestinal BD who underwent surgery due to bowel complications and underwent follow-up colonoscopy were retrospectively investigated. Their clinical data, including colonoscopic findings, were retrieved. Classification and regression tree analysis was used to develop an appropriate endoscopic classification model that can explain the postsurgical recurrence of intestinal BD most accurately based on the following classification: e0, no lesions; e1, solitary ulcer <20 mm in size; e2, solitary ulcer $\geq 20 \mathrm{~mm}$ in size; and e3, multiple ulcers regardless of size. Results: Clinical relapse occurred in 37 patients (68.5\%). Among 38 patients with colonoscopic recurrence, only 29 patients had clinically relapsed. Multivariate analysis identified higher disease activity index for intestinal BD at colonoscopy (hazard ratio [HR], 1.013; 95\% confidence interval [Cl], 1.005 to 1.021; $\mathrm{p}=0.002)$ and colonoscopic recurrence $(\mathrm{HR}, 2.829 ; 95 \% \mathrm{Cl}$, 1.223 to $6.545 ; p=0.015$ ) as independent risk factors for clinical relapse of intestinal BD. Endoscopic findings were classified into four groups, and multivariate analysis showed that the endoscopic score was an independent risk factor of clinical relapse $(p=0.012)$. The risk of clinical relapse was higher in the e3 group compared to the e0 group (HR, 6.284; 95\% Cl, 2.036 to 19.391; $p=0.001)$. Conclusions: This new endoscopic scoring system could predict clinical relapse in patients after surgical resection of intestinal BD. (Gut Liver 2018;12:674-681)

Key Words: Behcet syndrome; Postoperative care; Recurrence; Endoscopy

\section{INTRODUCTION}

Behçet's disease (BD) is a multisystemic vasculitic disorder featuring multi-organ involvement including the gastrointestinal tract. ${ }^{1,2}$ According to the current criteria, "intestinal BD" can be diagnosed if there is a typical oval-shaped large ulcer in the terminal ileum or inflammation in the small or large intestine with clinical findings meeting the diagnostic criteria of $\mathrm{BD}^{3,4}$ The clinical disease course of intestinal BD may be difficult to predict, as it can range from quiescent disease to chronic refractory disease that can result in complications such as intestinal perforation, fistula, or massive intestinal bleeding, eventually requiring surgical treatment. ${ }^{5}$ Ulcers in intestinal $\mathrm{BD}$ tend to perforate at multiple sites and since optimal treatment strategies remain largely unknown, surgery is often performed for patients unresponsive to medical treatments or those with bowel complications. ${ }^{6}$ More importantly, clinical recurrence of intestinal $\mathrm{BD}$ after bowel resection is frequent, as reported by our previous studies; cumulative surgery rates of intestinal BD were 6.7\% and $15.1 \%$ at 2 and 5 years after diagnosis, respectively, and the recurrence rate of intestinal lesions was approximately 50\% at 2 years postoperatively. ${ }^{7}$ However, demographic or clinical risk factors and endoscopic findings at follow-up that are highly predictive of postoperative recurrence or recrudescence of symptomatic intestinal BD have not yet been clearly identified.

Endoscopic recurrence correlates with the subsequent development of clinical recurrence as well as the development of complications and need for reoperation. Since endoscopic findings such as a typically shaped ulcer in the ileocecal region or at the anastomotic site are critical for diagnosis along with defining recurrence of intestinal $\mathrm{BD},{ }^{3,4}$ a regular follow-up endoscopy is essential after intestinal surgery. Rutgeerts et al. ${ }^{8}$ have developed an endoscopic scoring system for Crohn's disease (CD) that is classified by the number and distribution of ulcerations

Correspondence to: Jae Hee Cheon

Department of Internal Medicine, Yonsei University College of Medicine, 50-1 Yonsei-ro, Seodaemun-gu, Seoul 03722, Korea

Tel: +82-2-2228-1990, Fax: +82-2-393-6884, E-mail: GENIUSHEE@yuhs.ac

Received on December 4, 2017. Revised on April 1, 2018. Accepted on April 27, 2018. Published online September 21,2018 pISSN 1976-2283 eISSN 2005-1212 https://doi.org/10.5009/gnl17547

@ This is an Open Access article distributed under the terms of the Creative Commons Attribution Non-Commercial License (http://creativecommons.org/licenses/by-nc/4.0) which permits unrestricted non-commercial use, distribution, and reproduction in any medium, provided the original work is properly cited. 
at follow-up endoscopy and can predict postoperative recurrence of CD. Moreover, Schwartz and Regueiro ${ }^{9}$ have suggested a treatment algorithm which outlines management approaches based on the risk of recurrence in $\mathrm{CD}$. However, there are no reports of an endoscopic scoring system or treatment algorithm after surgery in intestinal BD.

Therefore, this study aimed to develop an appropriate scoring system using follow-up endoscopic findings which can predict recurrence of intestinal BD after intestinal resection.

\section{MATERIALS AND METHODS}

\section{Study subjects and diagnostic criteria}

Intestinal BD patients who underwent surgical intervention due to refractoriness to medical treatment or development of complications from intestinal BD at Severance Hospital, Yonsei University College of Medicine, Seoul, Korea, between March 1986 and December 2015 were retrospectively identified. Intestinal BD was diagnosed according to established criteria based on colonoscopic features and clinical manifestations. ${ }^{3,4}$ Patients classified as "definite," "probable," and "suspected" were included in the study. We excluded suspected patients with any evidence of another gastrointestinal disease such as CD, intestinal tuberculosis, or ischemic colitis during the follow-up period.

\section{Clinical evaluations and definition of variables}

The patient medical records were reviewed by two physicians (J.H.C. and J.W.P.). Among them, 54 patients who underwent at least one follow-up endoscopy within 5 years after surgery were selected, regardless of clinical relapse. After selection, demographic and preoperative clinical data including age at diagnosis, prior abdominal surgical history due to intestinal BD, gastrointestinal symptoms at diagnosis, and disease activity index prior to surgery and at the time of follow-up colonoscopy were retrieved. Systemic manifestations, clinical BD subtypes, follow-up colonoscopic findings (ulcer location, distribution, shape, number, and depth) and history of perioperative drug usage were retrieved. Since there are very limited medication options for intestinal BD, clinicians usually maintain previous medications used before surgery even after the bowel resection. The term "perioperative" is used for this reason. We also evaluated laboratory results including leukocyte counts, hemoglobin level, erythrocyte sedimentation rate, C-reactive protein level, and albumin level at the time of follow-up colonoscopy.

We measured intestinal BD activity using the disease activity index for intestinal BD (DAIBD). The DAIBD provides a total score drawn from eight variables (general well-being, fever, extra-intestinal manifestations, abdominal pain, abdominal mass, abdominal tenderness, intestinal complications, and number of liquid stools), with a higher score reflecting greater disease activity. Patients with DAIBD scores $<19,20-39,40-74$, and $\geq 75$ were considered to have remission, mild, moderate, and severe disease activities, respectively. ${ }^{10}$ Clinical relapse was defined as DAIBD $\geq 40$, existence of newly added medications, admission due to symptomatic aggravation, or re-operation related to intestinal BD. As for the colonoscopy findings, the location, shape (oval, geographic, or volcano type), distribution (localized or diffuse), number, depth (aphthous, shallow or deep) and size ( $<10 \mathrm{~mm}, 10$ to $20 \mathrm{~mm}$ or $\geq 20 \mathrm{~mm}$ ) of intestinal ulcers were described. If any newly developed ulcer was found in postoperative bowel analysis, the patient was considered to have endoscopically recurrent disease. Symptomatic relapse was defined when the patient expressed an intestinal symptom or symptoms at the time of follow-up colonoscopy.

This study was approved by the Institutional Review Board of Severance Hospital, Seoul, Korea (IRB number: 4-2017-2022).

\section{Statistical analysis}

The software program SPSS version 21.0 (IBM Corp., Armonk, NY, USA) was used for statistical analyses. Student t-test or the Mann-Whitney U-test was used to compare numerical variables between groups. The chi-square or Fisher exact test was used to compare categorical variables. $p$-values $<0.05$ were considered statistically significant. Classification and regression tree (CART) analysis ${ }^{11,12}$ was used to determine an appropriate model of endoscopic classification which can explain the postsurgical recurrence of intestinal BD most accurately.

CART is a decision tree algorithm developed by Breiman et al. ${ }^{11}$ in 1984. CART algorithm can be used for building both "classification" and "regression" decision trees. The impurity (or purity) measure used in building decision tree in CART is "Gini Index." In this tree building, choosing the best splitter is to minimize the average "impurity" of the two-child nodes and based on this, the first split must be whether colonoscopic recurrence has occurred or not. After that, the second split could be any other factor; however, depth and size could be correlated as larger ulcer is mostly deeper in depth. For this reason, we selected the size factor rather than the depth factor, which could be more objective. Shape of the recurred ulcer was another important factor, but in most cases, they were oval-shaped. Only two cases had volcano-shaped ulcer and we decided that this could not result in accurate analysis, for the total case number was small (54 cases). Accordingly, factors left are the number and size of ulceration. Derived from Gini Index, best tree resulted in the second and third split to be the number and then the size of ulcer. This resulted in the new following endoscopic scoring model; e0, no lesions; e1, <20 mm sized solitary ulcer; e2, $\geq 20$ mm sized solitary ulcer; and e3, multiple ulcers regardless of size.

We also constructed multivariate Cox regression models to identify independent factors associated with severe clinical disease course. Adjusted odds ratios and 95\% confidence intervals (CIs) were calculated. 


\section{RESULTS}

\section{Baseline clinical and endoscopic characteristics of pa- tients with intestinal BD}

A total of 91 intestinal BD patients who underwent surgical intervention due to intestinal BD were identified. Thirty-six patients were excluded due to incomplete data because of lack of colonoscopic or clinical data. One patient was excluded due to follow-up loss. The remaining 54 patients were eligible for this study with 28 male and 26 female patients. Clinical disease relapse occurred in 37 patients (68.5\%) and of these, 16 patients (29.6\%) underwent re-operation. Thirty-eight patients (70.37\%) had colonoscopic recurrence at follow-up colonoscopy and of these, only 29 had clinical relapse.

Clinical characteristics and demographics of patients are listed in Table 1 . The median age at diagnosis was 36 years (range,

Table 1. Comparison of Baseline Characteristics of Two Groups According to Clinical Relapse

\begin{tabular}{lc}
\hline \multicolumn{1}{c}{ Characteristics } & $\begin{array}{c}\text { Total patients } \\
(\mathrm{n}=54)\end{array}$ \\
\hline Age at diagnosis, yr & $36(12-64)$ \\
Male sex & $28(51.9)$ \\
Disease duration, mo & $126.5(21-432)$ \\
Clinical subtype & \\
Complete & $37(68.5)$ \\
Incomplete & $17(31.5)$ \\
Suspected & 0 \\
Systemic symptoms and signs of BD at surgery & \\
Recurrent oral ulcer & $35(64.8)$ \\
Recurrent genital ulcer & $15(27.8)$ \\
Ocular lesion & $3(5.5)$ \\
Skin lesion & $15(27.8)$ \\
Positive pathergy test & $1(1.8)$ \\
Arthritis or arthralgia & $14(25.9)$ \\
Neurologic lesion & 0 \\
Vascular lesion & 0 \\
History of previous abdominal surgery & $22(40.7)$ \\
Medication history before surgery & $30(70.45)$ \\
Immunomodulator & \\
Anti-TNF- $\alpha$ antibody & $31(57.4)$ \\
Corticosteroid & $21.67 \pm 34.29$ \\
Disease activity index for intestinal & \\
BD at surgery & \\
Disease activity index for intestinal & \\
BD at follow-up & \\
\hline
\end{tabular}

Data are presented as median (range) or number (\%). $\mathrm{BD}$, Behçet’s disease; TNF, tumor necrosis factor.
12 to 64 years) and median disease duration was 126.5 months (range, 21 to 432 months). Thirty-seven patients (68.5\%) were complete type of intestinal BD and most of them had recurrent oral ulcer (64.8\%). Twenty-two (40.7\%) had history of at least one time of intestinal resection before the last surgery. Thirtyone (57.4\%) were using immunomodulators before surgery and $87.0 \%$ of total patients were on systemic steroids. The mean DAIBD score before surgery was checked as $121.67( \pm 34.29)$, but was decreased to $45.74( \pm 36.90)$ after surgery. The endoscopic characteristics at follow-up colonoscopy are listed in Table 2. All colonoscopically recurred cases had ulcers located in the anastomosis site and 35 cases (92.1\%) occurred in the localized area. Regarding the depth and appearance of intestinal ulcers, 12 patients (31.6\%) had well-demarcated deep ulcers considered typical of intestinal BD, 26 patients (68.4\%) had oval-shaped ulcers, 10 patients (26.3\%) had geographic ulcers, and two patients (5.3\%) had volcano-type ulcers.

\section{Risk factors for clinical relapse after surgery in intestinal BD patients}

The main aim of this study was to establish an appropriate scoring system using follow-up endoscopic findings that can predict clinical recurrence after surgical resection of an affected bowel in intestinal BD patients. Therefore, Kaplan-Meier curves were constructed to compare relapse-free survival of patients classified by clinical and endoscopic factors including colonoscopic recurrence. Based on this analysis, only colonoscopic recurrence was associated with the development of clinical relapse (hazard ratio [HR], 2.829; 95\% CI, 1.223 to 6.545; $p=0.015$ ) (Fig.

Table 2. Colonoscopic Findings in 38 Intestinal Behçet's Disease Patients with Colonoscopic Recurrence

\begin{tabular}{lc}
\hline & No. $(\%)$ \\
\hline Site of recurrence & $38(100.0)$ \\
Anastomosis site & 0 \\
Other site & \\
Distribution & $35(92.1)$ \\
Localized & $3(7.9)$ \\
Diffuse & \\
Depth & $9(23.7)$ \\
Aphthous & $17(44.7)$ \\
Shallow & $12(31.6)$ \\
Deep & \\
Shape & $26(68.4)$ \\
Oval & $10(26.3)$ \\
Geographic & $2(5.3)$ \\
Volcano & \\
Size, mm & $25(65.8)$ \\
$<20$ & $13(34.2)$ \\
$\geq 20$ &
\end{tabular}


1A). Younger age at diagnosis ( $\leq 35$ years), history of immunomodulator or perioperative anti-tumor necrosis factor- $\alpha$ (antiTNF- $\alpha$ ) antibody usage were not associated with the development of clinical relapse (Fig. 1B-D).

Univariate and multivariate Cox regression analysis adjusting for age at diagnosis, sex and disease duration were performed. This identified two independent risk factors associated with clinical relapse; higher DAIBD at colonoscopy (HR, 1.013; 95\% CI, 1.005 to $1.021 ; \mathrm{p}=0.002)$ and colonoscopic recurrence (HR, 2.829; $95 \%$ CI, 1.223 to $6.545 ; \mathrm{p}=0.015$ ) were independent risk factors for clinical relapse in intestinal BD (Table 3). Perioperative immu-

\section{A}

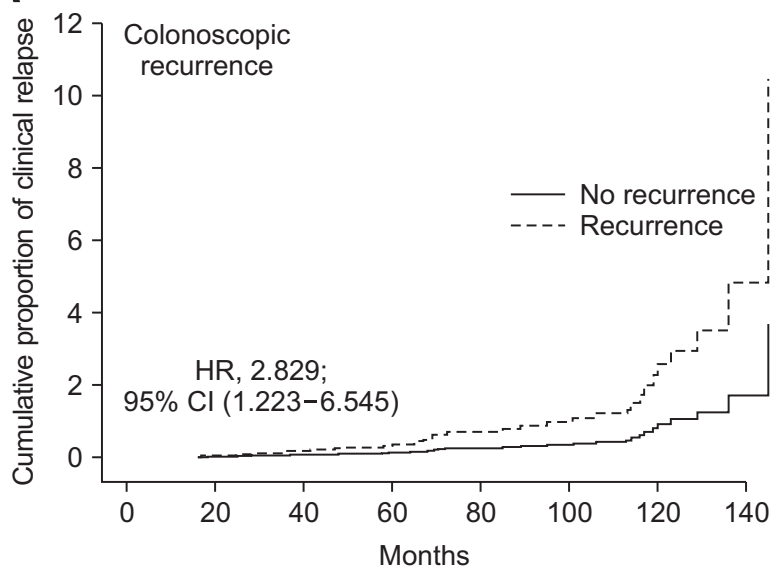

\begin{tabular}{lcccc}
\hline $\begin{array}{c}\text { No. of } \\
\text { patients at risk }\end{array}$ & 0 mo & 40 mo & 80 mo & 120 mo \\
\hline No recurrence & 16 & 14 & 7 & 4 \\
Recurrence & 38 & 27 & 16 & 5 \\
\hline
\end{tabular}

\section{C}

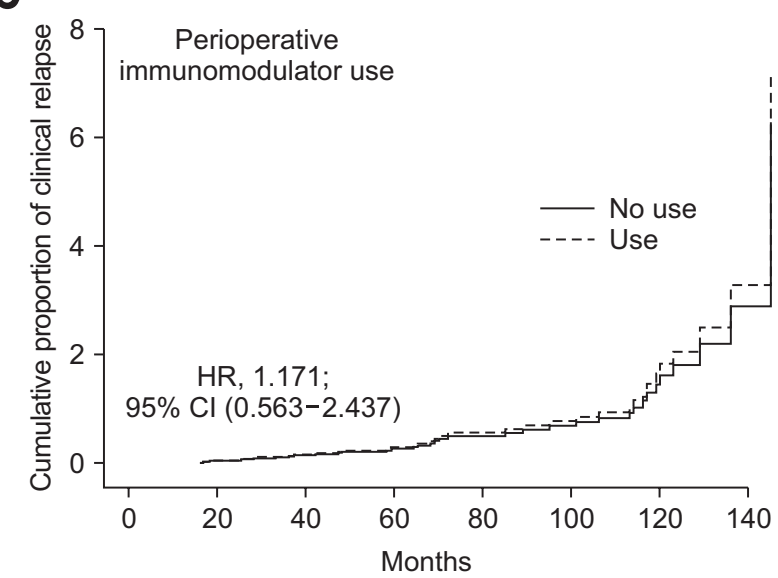

\begin{tabular}{ccccc}
\hline $\begin{array}{c}\text { No. of } \\
\text { patients at risk }\end{array}$ & 0 mo & 40 mo & 80 mo & 120 mo \\
\hline No use & 16 & 13 & 8 & 4 \\
Use & 38 & 28 & 15 & 5 \\
\hline
\end{tabular}

nomodulator usage did not affect clinical relapse, as well as perioperative steroids or anti-TNF- $\alpha$ antibody usage. Total follow-up duration was median 26.5 months (range, 1 to 165 months).

We performed another multivariate analysis to determine which colonoscopic findings of recurred ulceration were associated with clinical relapse (Table 4). The number of ulcerations, whether solitary or multiple, was significantly associated with clinical relapse (HR, 2.884; 95\% CI, 1.038 to 8.013; $p=0.042$ ). The size of the recurred ulceration also showed a relation to higher clinical relapse, although this was not a statistically significant result.

B

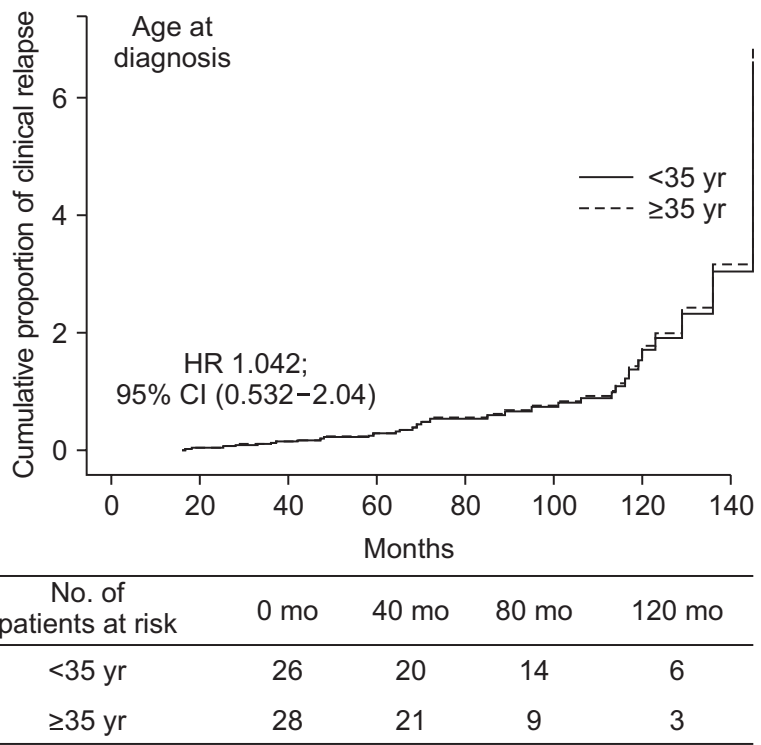

D

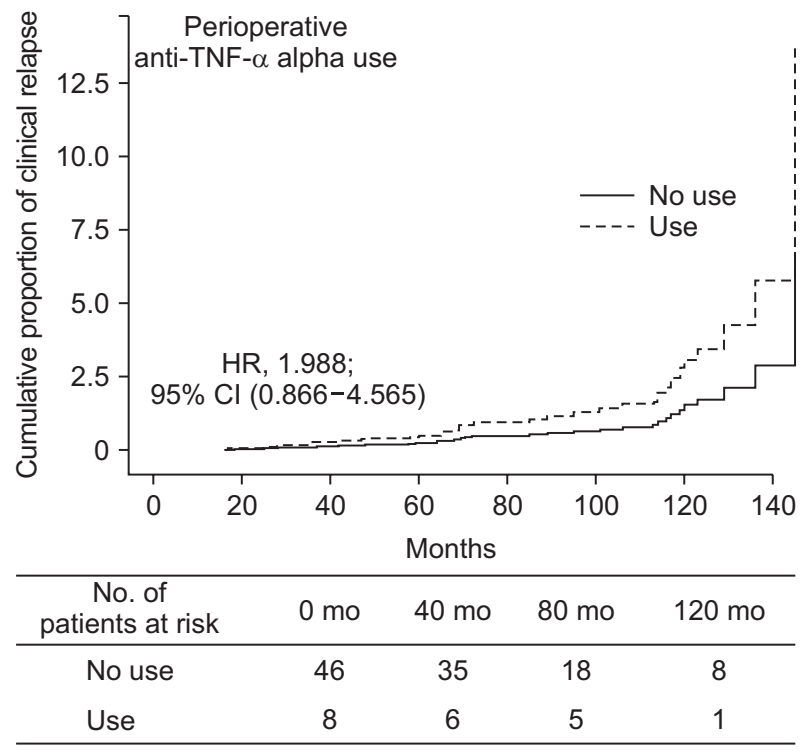

Fig. 1. Clinical relapse-free survival rate during long-term follow-up in intestinal Behçet's disease patients grouped according to clinical factors colonoscopic recurrence (A), age at diagnosis (B), perioperative immunomodulator usage (C), perioperative anti-tumor necrosis factor- $\alpha$ antibody usage (D).

HR, hazard ratio; CI, confidence interval. 
Table 3. Cox Hazard Regression Analysis of Risk Factors for Clinical Relapse after Intestinal Resection

\begin{tabular}{|c|c|c|c|c|}
\hline & Crude OR (95\% CI) & p-value & Adjusted OR (95\% CI)* & p-value \\
\hline DAIBD at initial diagnosis & $1.003(0.993-1.013)$ & 0.549 & $1.001(0.991-1.011)$ & 0.904 \\
\hline DAIBD at follow-up colonoscopy & $1.006(0.999-1.013)$ & 0.073 & $1.013(1.005-1.021)$ & 0.002 \\
\hline Perioperative immunomodulator use (no vs yes) & $1.051(0.526-2.102)$ & 0.887 & $1.171(0.563-2.437)$ & 0.673 \\
\hline Perioperative anti-TNF- $\alpha$ antibody use (no vs yes) & $1.565(0.702-3.489)$ & 0.274 & $1.988(0.866-4.565)$ & 0.105 \\
\hline Perioperative steroid use (no vs yes) & $1.196(0.415-3.442)$ & 0.741 & $2.453(0.742-8.111)$ & 0.141 \\
\hline Colonoscopic recurrence (no vs yes) & $1.747(0.795-3.848)$ & 0.165 & $2.829(1.223-6.545)$ & 0.015 \\
\hline
\end{tabular}

OR, odds ratio; CI, confidence interval; DAIBD, disease activity index for intestinal Behçet's disease; TNF, tumor necrotic factor.

*Adjusted for sex, age at diagnosis, disease duration.

Table 4. Multivariate Cox Hazard Regression Analysis of Colonoscopic Findings for Clinical Relapse after Intestinal Resection

\begin{tabular}{llc}
\hline \multicolumn{1}{c}{ HR (95\% CI) } & p-value \\
\hline Ulcer description & & \\
Location (ileocecal area vs others) & $0.908(0.208-3.96)$ & 0.898 \\
Distribution (localized vs diffuse) & $0.553(0.116-2.643)$ & 0.458 \\
Depth & & 0.507 \\
Aphthous vs shallow & $1.500(0.484-4.643)$ & 0.482 \\
Aphthous vs deep & $0.464(0.042-5.134)$ & 0.531 \\
Shape & & 0.184 \\
Oval vs geographic & $0.369(0.125-1.096)$ & 0.073 \\
Oval vs volcano & $0.678(0.083-5.545)$ & 0.717 \\
Size ( $\leq 20$ mm vs $>20$ mm) & $5.030(0.469-53.914)$ & 0.182 \\
Number (solitary vs multiple) & $2.884(1.038-8.013)$ & 0.042 \\
\hline
\end{tabular}

HR, hazard ratio; CI, confidence interval.

\section{Development of an endoscopic scoring system to predict relapse after surgery in intestinal BD patients}

Endoscopic findings needed to be classified properly to determine an appropriate model to best predict postsurgical recurrence of intestinal BD. Hence, we used the CART analysis (Fig. 2). Among 54 patients, 38 patients showed colonoscopic findings at follow-up which implied recurrence and were classified into another two groups based on the number of ulcerations: multiple $(n=15)$ versus solitary $(n=23)$. Fifteen patients had multiple ulcerations and of these, 12 patients (80.0\%) were clinically relapsed. Twenty-three patients with solitary ulcers were divided into the next two groups by size of ulceration: shorter than 20 $\mathrm{mm}$ in diameter $(\mathrm{n}=13)$ versus $20 \mathrm{~mm}$ or longer in diameter $(\mathrm{n}=10)$. Patients with small ulcers had a lower relapse rate $(9 / 13$, $69.2 \%$ ), while patients with a larger ulcer had a higher relapse rate $(8 / 10,80.0 \%)$. Based on this result, endoscopic findings were classified into four groups according to the size and number of recurred ulcer(s); e0, no lesions; e1, <20 mm sized solitary ulcer; e2, $\geq 20 \mathrm{~mm}$ sized solitary ulcer; and e3, multiple ulcers regardless of size.

Multivariate analysis adjusting for sex, age at diagnosis, disease duration, previous surgical resection, perioperative medica- tion usage and disease activity score showed that the endoscopic score was an independent risk factor of clinical relapse ( $p=0.012)$. The risk of clinical relapse was approximately 6 times higher in the e3 colonoscopy group compared to the e0 group (HR, 6.284; 95\% CI, 2.036 to 19.391; $\mathrm{p}=0.001$ ) (Fig. 3). The e2 group (HR, $1.953 ; 95 \% \mathrm{CI}, 0.545$ to $6.997 ; \mathrm{p}=0.304)$ and the e1 group (HR, 1.672 ; $95 \%$ CI, 0.519 to $5.39 ; \mathrm{p}=0.389$ ) also showed numerically higher risk of clinical relapse than the e0 group, while it did not show any statistical significance.

\section{DISCUSSION}

Intestinal BD is characterized by deep ulcers, which are most commonly located in the terminal ileum or the ileocecal region and tend to perforate or penetrate the intestinal wall. ${ }^{13,14}$ Therefore, a considerable number of patients with intestinal BD eventually require emergency or elective operations and likewise, patients who have little or no response to medical treatment often undergo surgical intervention., ${ }^{6,15}$ This disease is also well known for a high risk of early recurrence after intestinal resection. ${ }^{16-18}$ Previous studies including ours showed that the recurrence rate of intestinal lesions was over 50\% at 2 years postoperatively and as high as 75\% in one study., ${ }^{7,19}$ To our knowledge, there are few preceding studies investigating the prediction of postoperative recurrence of intestinal BD. Furthermore, there have been no studies on the development of an objective endoscopic scoring system to predict the postoperative recurrence of intestinal BD. Our current study is the first to present an actual endoscopic scoring system to predict clinical relapse of intestinal BD.

Rutgeerts et al. ${ }^{8}$ developed an endoscopic scoring system related to the postoperative recurrence of $\mathrm{CD}$. This was a prospective endoscopic cohort study which demonstrated that the postoperative clinical course of $\mathrm{CD}$ is best predicted by the severity of endoscopic lesions of $\mathrm{CD}$ during the first year after resection. They also examined the natural history of $\mathrm{CD}$ recurrence in other studies, which consistently showed that the severity of endoscopic recurrence at 1 year is predictive of earlier clinical relapse. These studies regarding endoscopic prediction of postoperative recurrence prompted numerous sequential studies.

Similarly, in this study, we compared five colonoscopic pa- 


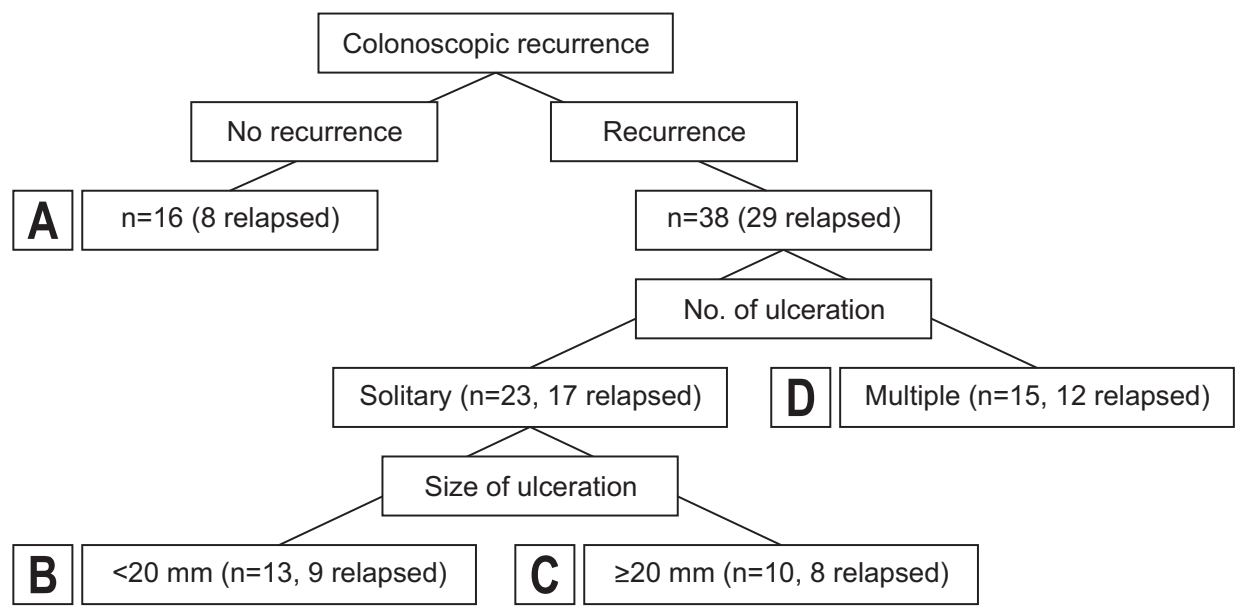

\begin{tabular}{ccr}
\hline \multirow{2}{*}{ Terminal nodes } & \multicolumn{2}{c}{ Clinical relapse, no. $(\%)$} \\
\cline { 2 - 3 } & No relapse & Relapse \\
\hline A & $8(50.0)$ & $8(50.0)$ \\
B & $4(30.8)$ & $9(69.2)$ \\
C & $2(20.0)$ & $8(80.0)$ \\
D & $3(20.0)$ & $12(80.0)$ \\
\hline
\end{tabular}

Fig. 2. Classification and regression tree analysis.

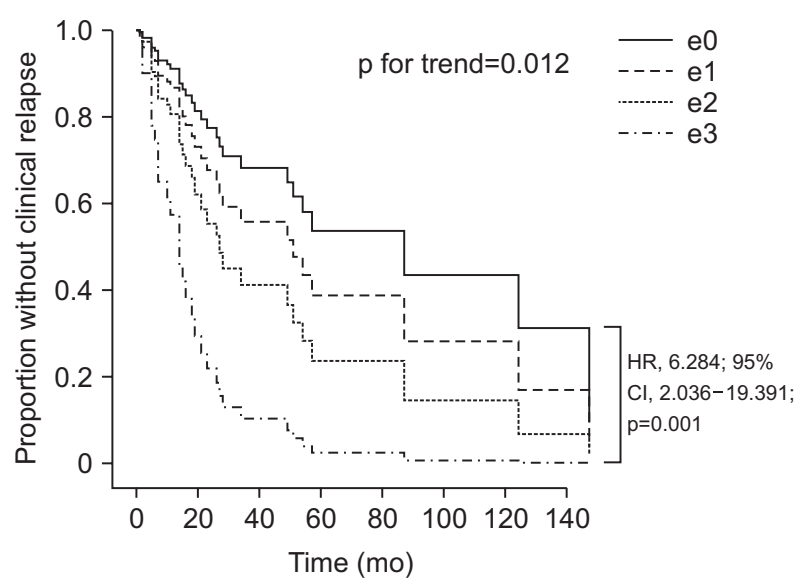

\begin{tabular}{ccccc}
\hline $\begin{array}{c}\text { No. of } \\
\text { patients at risk }\end{array}$ & 0 mo & 40 mo & 80 mo & 120 mo \\
\hline E0 & 15 & 14 & 7 & 4 \\
E1 & 12 & 8 & 4 & 0 \\
E2 & 9 & 8 & 7 & 3 \\
E3 & 14 & 11 & 5 & 2 \\
\hline
\end{tabular}

Fig. 3. Actuarial analysis of clinical relapse in patients stratified according to the severity of endoscopic lesions at follow-up endoscopy. $\mathrm{HR}$, hazard ratio; CI, confidence interval. Median follow-up period: 26.5 months (range, 1-165 months).

rameters and adopted the CART method ${ }^{12}$ as an alternative to generate a simpler and easier scoring system than the diagnostic tools derived from logistic regression. The CART method identified the number and size of ulcers as the most distinguishable features. By the sequential application of these two variables, with ulcer number followed by lesion size, we developed a novel endoscopic scoring system composed of four categories. As demonstrated by our results, an endoscopic score of "e3" which represented the group with multiple ulcers regardless of size, had a 6.284 higher chance of recurrence than the group with no lesions. This result suggests an applicable parameter which can be useful to foresee the postoperative clinical recurrence of intestinal $\mathrm{BD}$, and can also possibly be used to develop a strategy for future treatment of recurrent intestinal BD after bowel resection surgery.

Interestingly, a similar result was obtained in the separate multivariate analysis of endoscopic features of recurred ulcersthe number of ulcerations, whether solitary or multiple, was significantly associated with clinical relapse (HR, 2.884; 95\% CI, 1.038 to $8.013 ; \mathrm{p}=0.042$ ) which is a novel finding. According to other previous studies including ours, volcano-shaped ulcers were usually identified as an independent predictive factor for poor prognosis and recurrence. ${ }^{7,19-21} \mathrm{Kim}$ et al. ${ }^{22}$ indicated that volcano-type ulcerations in intestinal BD demonstrated a significantly lower remission rate with medical treatment, higher cumulative surgery rate, and higher recurrence rate. Jung et al. ${ }^{20}$ reported that volcano-type ulcers are also predictive factors for recurrence and reoperation in patients treated surgically. These findings may explain the high surgery rate of intestinal BD patients, along with another study which demonstrated that patients with volcano-shaped ulcers have an increased risk of free bowel perforation. ${ }^{23}$ On the other hand, there was one Japanese study reporting that the number of ulcerations was related to recurrence, relapse and perforation. ${ }^{15}$ Why multiplicity of a 
recurrent ulcer is inclined to more frequent clinical relapse is still unknown; however, this can be somewhat explained by the fact that mucosal healing is significantly associated with clinical courses of various inflammatory bowel diseases. Since Rutgeerts et al. ${ }^{24}$ proposed to focus on mucosal healing and disease course as primary outcome measures, studies regarding mucosal healing and prognosis of inflammatory bowel diseases have been consistently accumulating. A Norwegian study reported that mucosal healing after 1 year of treatment is predictive of reduced subsequent disease activity and decreased need for active treatment. ${ }^{25}$ Moreover, one of our studies also demonstrated that mucosal healing is an independent factor predictive of the long-term prognosis of intestinal $\mathrm{BD} .^{26}$ From these data, we can carefully conjecture that the amount of defected mucosa might be associated with a higher recurrence rate and worse prognosis, so patients with multiple ulcers might be more prone to clinical relapse compared to patients with a solitary ulcer. These results not only give further strength to the use of mucosal healing as a clinical indicator and treatment goal in intestinal BD, but also suggest another candidate for future study-the relationship between total surface area of a mucosal defect and clinical prognosis.

Our results also showed that a higher DAIBD score at followup colonoscopy was associated with a higher rate of clinical relapse. The DAIBD is composed of eight items. This finding suggests that the DAIBD score should be essential along with the colonoscopic scoring system in creating a treatment algorithm to predict recurrent intestinal BD.

In our study, all cases of the recurrent ulcers were noted at the anastomotic site and were mostly localized. Furthermore, the median relapse-free survival was 14 months and ranged from 1 to 147 months. Jung et al. ${ }^{20}$ also reported that the cumulative recurrence rate after surgical treatment was $29.2 \%$ at 2 years and $47.2 \%$ at 5 years. Therefore, it is suggested that in patients with intestinal BD who undergo surgery, follow-up examinations with radiography and endoscopy should begin before 2 years postoperatively with careful focus on the anastomotic site.

This study has several limitations. First, the number of patients was insufficient to reach a decisive conclusion, even though our study results were statistically eligible. Accordingly, this system should be validated in a larger scale, prospective cohort. Second, we retrospectively analyzed clinical and colonoscopic characteristics and postoperative follow-up outcomes when determining the likelihood of clinical relapse. Moreover, follow-up endoscopies were performed at different time points after surgery.

In conclusion, this new endoscopic scoring system might help predict clinical relapse in patients undergoing surgical resection of intestinal BD. Furthermore, as stated above, this system could be the basis for developing an algorithm concerning the treatment of intestinal BD after surgery.

\section{CONFLICTS OF INTEREST}

No potential conflict of interest relevant to this article was reported.

\section{ACKNOWLEDGEMENTS}

This research was supported by a grant (A120176) from the Korean Health Technology R\&D Project through the Korea Health Industry Development Institute (KHIDI), which is funded by the Ministry of Health and Welfare, Republic of Korea; a grant (NRF-2017R1A1A1A05001011) from the Basic Science Research Program through the National Research Foundation of Korea, which is funded by the Ministry of Science, ICT and Future Planning; and a faculty research grant (2012-31-0477) from the Department of Internal Medicine, Yonsei University College of Medicine.

\section{REFERENCES}

1. Kaklamani VG, Vaiopoulos G, Kaklamanis PG. Behçet's disease. Semin Arthritis Rheum 1998;27:197-217.

2. Sakane T, Takeno M, Suzuki N, Inaba G. Behçet’s disease. N Engl J Med 1999;341:1284-1291.

3. Cheon JH, Kim ES, Shin SJ, et al. Development and validation of novel diagnostic criteria for intestinal Behçet's disease in Korean patients with ileocolonic ulcers. Am J Gastroenterol 2009;104:2492-2499.

4. Kobayashi K, Ueno F, Bito S, et al. Development of consensus statements for the diagnosis and management of intestinal Behçet's disease using a modified Delphi approach. J Gastroenterol 2007;42:737-745.

5. Kurata M, Nozue M, Seino K, Murata H, Sumita T, Katashi F. Indications for surgery in intestinal Behçet's disease. Hepatogastroenterology 2006;53:60-63.

6. Ketch LL, Buerk CA, Liechty D. Surgical implications of Behçet's disease. Arch Surg 1980;115:759-760.

7. Jung YS, Cheon JH, Park SJ, et al. Clinical course of intestinal Behcet's disease during the first five years. Dig Dis Sci 2013;58:496503.

8. Rutgeerts P, Geboes K, Vantrappen G, Beyls J, Kerremans R, Hiele M. Predictability of the postoperative course of Crohn's disease. Gastroenterology 1990;99:956-963.

9. Schwartz M, Regueiro M. Prevention and treatment of postoperative Crohn's disease recurrence: an update for a new decade. Curr Gastroenterol Rep 2011;13:95-100.

10. Cheon JH, Han DS, Park JY, et al. Development, validation, and responsiveness of a novel disease activity index for intestinal Behçet's disease. Inflamm Bowel Dis 2011;17:605-613.

11. Breiman L, Friedman J, Olshen RA, Stone CJ. Classification and regression trees. New York: Chapman and Hall, 1984.

12. Steinberg D, Colla P. CART: Classification And Regression Trees. 
San Diego: Salford Systems, 1997.

13. Bayraktar Y, Ozaslan E, Van Thiel DH. Gastrointestinal manifestations of Behcet's disease. J Clin Gastroenterol 2000;30:144-154.

14. Ebert EC. Gastrointestinal manifestations of Behçet’s disease. Dig Dis Sci 2009;54:201-207.

15. Kasahara Y, Tanaka S, Nishino M, Umemura H, Shiraha S, Kuyama T. Intestinal involvement in Behçet's disease: review of 136 surgical cases in the Japanese literature. Dis Colon Rectum 1981;24:103-106.

16. Baek SJ, Kim CW, Cho MS, et al. Surgical treatment and outcomes in patients with intestinal Behçet disease: long-term experience of a single large-volume center. Dis Colon Rectum 2015;58:575-581.

17. Choi IJ, Kim JS, Cha SD, et al. Long-term clinical course and prognostic factors in intestinal Behçet's disease. Dis Colon Rectum 2000;43:692-700.

18. Iida M, Kobayashi H, Matsumoto T, et al. Postoperative recurrence in patients with intestinal Behçet’s disease. Dis Colon Rectum 1994;37:16-21.

19. Naganuma M, Iwao $\mathrm{Y}$, Inoue N, et al. Analysis of clinical course and long-term prognosis of surgical and nonsurgical patients with intestinal Behçet's disease. Am J Gastroenterol 2000;95:28482851.

20. Jung YS, Yoon JY, Lee JH, et al. Prognostic factors and long-term clinical outcomes for surgical patients with intestinal Behcet's disease. Inflamm Bowel Dis 2011;17:1594-1602.

21. Chung MJ, Cheon JH, Kim SU, et al. Response rates to medical treatments and long-term clinical outcomes of nonsurgical patients with intestinal Behçet disease. J Clin Gastroenterol 2010;44:e116-e122.

22. Kim JS, Lim SH, Choi IJ, et al. Prediction of the clinical course of Behçet's colitis according to macroscopic classification by colonoscopy. Endoscopy 2000;32:635-640.

23. Moon CM, Cheon JH, Shin JK, et al. Prediction of free bowel perforation in patients with intestinal Behçet's disease using clinical and colonoscopic findings. Dig Dis Sci 2010;55:2904-2911.

24. Rutgeerts P, Vermeire S, Van Assche G. Mucosal healing in inflammatory bowel disease: impossible ideal or therapeutic target? Gut 2007;56:453-455.

25. Frøslie KF, Jahnsen J, Moum BA, Vatn MH; IBSEN Group. Mucosal healing in inflammatory bowel disease: results from a Norwegian population-based cohort. Gastroenterology 2007;133:412422.

26. Yim SM, Kim DH, Lee HJ, et al. Mucosal healing predicts the long-term prognosis of intestinal Behçet's disease. Dig Dis Sci 2014;59:2529-2535. 